\title{
DETERMINANTS, CHOICES AND COMBINATORICS
}

\author{
J. MALKOUN
}

\begin{abstract}
We prove a formula which generalizes both Onn's colorful determinantal formula, related to Rota's basis conjecture, and Svrtan's $n$ ! formula, related to the Atiyah-Sutcliffe problem. In some cases, our formula allows us to prove some results similar in spirit to the statement of Rota's basis conjecture. We prove such a result using Svrtan's $n$ ! formula, generalizing one of Svrtan's arguments to a combinatorial setting.
\end{abstract}

\section{Introduction}

In 1989, Rota conjectured his famous basis conjecture which, at the time of writing, is still open in general.

Theorem 1.1. Let $V$ be a finite-dimensional vector space over a field $\mathbb{F}$. Suppose, ${ }^{1} A, \ldots,{ }^{n} A$ are $n$ bases of $V$. Then for each $i$, there is a linear order of ${ }^{i} A$, say

$$
{ }^{1} A=\left\{{ }^{1} a_{1},{ }^{1} a_{2}, \ldots,{ }^{1} a_{n}\right\} ; \ldots ;{ }^{n} A=\left\{{ }^{n} a_{1},{ }^{n} a_{2}, \ldots,{ }^{n} a_{n}\right\},
$$

such that

$$
{ }_{1} C=\left\{{ }^{1} a_{1},{ }^{2} a_{1}, \ldots,{ }^{n} a_{1}\right\} ; \ldots ;{ }_{n} C=\left\{{ }^{1} a_{n},{ }^{2} a_{n}, \ldots,{ }^{n} a_{n}\right\}
$$

are $n$ bases.

The conjecture originated in Rota's work in invariant theory, and implies some identities in invariant theory and representation theory (cf. [13] and [14]). The literature surrounding Rota's basis conjecture is vast, and shall not be reviewed here. The reader may look for instance at [7, [8], [9], [10], [11], [12] and the references therein.

We do mention though S. Onn's article [14, where he proves his colorful determinantal formula, and uses it to show that the AlonTarsi conjecture on Latin squares implies Rota's basis conjecture for even $n$. The Alon-Tarsi conjecture on Latin squares states that if $n$ is even, then the number of even $n$-by- $n$ Latin squares is different from the number of odd $n$-by- $n$ Latin squares.

Date: Received: date / Accepted: date. 
The Atiyah-Sutcliffe problem on configurations of points ([2], [3] and [5]) is a geometric problem with roots in Physics ([6]). Indeed, in ([6]), Berry and Robbins propose a quantum-mechanical and geometric approach to the spin-statistics theorem, to be contrasted with the standard argument which relies on quantum field theory. Their discussion there was mostly for 2 or 3 identical particles. In order to extend their work to $n$ identical particles, they needed a certain equivariant map to exist. This led to the so-called Berry-Robbins problem.

Berry-Robbins Problem: Does there exist, for each $n$, a continuous map

$$
f_{n}: C_{n}\left(\mathbb{R}^{3}\right) \rightarrow U(n) / T^{n}
$$

from the configuration space $C_{n}\left(\mathbb{R}^{3}\right)$ of $n$ distinct points in $\mathbb{R}^{3}$ into the complete flag manifold $U(n) / T^{n}$, such that $f_{n}$ is equivariant under the action of the symmetric group $\Sigma_{n}$ ? More precisely, $\Sigma_{n}$ acts on a configuration of $n$ distinct points in $\mathbb{R}^{3}$ by permuting the $n$ points of that configuration, while it acts by permuting the columns of an element of $U(n)$, and this action induces an action on the quotient manifold $U(n) / T^{n}$.

In [2], Atiyah solved positively the Berry-Robbins problem, but the maps $f_{n}$ in that article were not very satisfactory: they were not smooth for instance. This led Atiyah to consider other candidate maps for $f_{n}$, which in turn led to the Atiyah-Sutcliffe conjectures, the area being sometimes referred to as the Atiyah-Sutcliffe problem on configurations of points. Consider $n$ distinct points $x_{i}$ in $\mathbb{R}^{3}$, for $1 \leq i \leq n$. To each pair $(i, j), 1 \leq i, j \leq n$ and $i \neq j$, form the unit vector $v_{i j}$ in the direction from $x_{i}$ to $x_{j}$, and then let $p_{i j}$ be the linear polynomial with root $v_{i j}$, after identifying the unit sphere of directions in $\mathbb{R}^{3}$ with the Riemann sphere using stereographic projection. For each $i$ between 1 and $n$, denote by $p_{i}$ the polynomial of degree less than $n$ defined by

$$
p_{i}=\prod_{j \neq i} p_{i j}
$$

The polynomials $p_{i}$ were conjectured by Atiyah in [2], 3] to be linearly independent over $\mathbb{C}$, for any given configuration $\left(x_{i}\right)$ of $n$ distinct points in $\mathbb{R}^{3}$. The conjecture was further refined by Atiyah together with Sutcliffe in [5], where two successively stronger conjectures were also made. In 2018, linear independence was proved in [4].

Svrtan in [15] considered not only the Atiyah-Sutcliffe determinant, relevant to the Atiyah-Sutcliffe conjectures, but also some modified 
determinant functions, corresponding to various subgraphs of the complete unoriented graph on $n$ vertices, with the Atiyah-Sutcliffe determinant being one of them. There he proved his $n$ ! formula, which in particular shows that, given any initial configuration of $n$ distinct points in $\mathbb{R}^{3}$, one of these modifed determinant functions must be nonzero.

In this article, we prove a formula (cf. Thm. 2.1) in section 2 generalizing both Onn's colorful determinantal formula in [14] and Svrtan's $n$ ! formula in [15], and show how to obtain these two formulas as special cases in sections 3 and 4 respectively. In the process, we also generalize Svrtan's argument to a combinatorial setting (cf. Thm. 4.1).

\section{An identity involving permutation groups and determinants}

In this section, we introduce a general formula which includes as special cases Onn's colorful determinantal formula, as well as a formula by Svrtan, and more. Let $\mathbb{F}$ be a fixed field. All matrices in this section will be assumed to have entries in the ground field $\mathbb{F}$. Given a square matrix $A$ of dimension $n$-by- $n$, we denote by $[A]$ the $n$-tuple of its columns, namely $[A]=\left(a_{1}, \cdots, a_{n}\right)$, where $a_{i}$ is the $i$-th column of $A$. Let ${ }^{1} A, \cdots,{ }^{k} A$ be $k$ square matrices of dimensions $n_{1}$-by- $n_{1}, \ldots, n_{k}$-by- $n_{k}$, respectively. Given the $k$-tuple of square matrices $\mathcal{A}=\left({ }^{1} A, \ldots,{ }^{k} A\right)$, we denote by

$$
[\mathcal{A}]=\left(\left[{ }^{1} A\right], \ldots,\left[{ }^{k} A\right]\right)
$$

Let

$$
\Sigma=\Sigma_{n_{1}} \times \cdots \times \Sigma_{n_{k}}
$$

where $\Sigma_{n}$ is the symmetric group on $[n]=\{1, \ldots, n\}$. There is a natural action of $\Sigma$ on the space of all $k$-tuples of square matrices as above. Namely, if $\sigma \in \Sigma$, we write $\sigma=\left(\sigma_{1}, \ldots, \sigma_{k}\right)$ where $\sigma_{i} \in \Sigma_{n_{i}}$, and then define

$$
\sigma \cdot \mathcal{A}=\left(\sigma_{1} \cdot\left({ }^{1} A\right), \ldots, \sigma_{k} \cdot\left({ }^{k} A\right)\right)
$$

where

$$
(\rho . A)_{i j}=A_{i \rho^{-1}(j)} \quad(1 \leq i, j \leq n)
$$

for any $n$-by- $n$ matrix $A$ and any $\rho \in \Sigma_{n}$. Thus we see that an element $\sigma \in \Sigma$ permutes the columns of each matrix ${ }^{i} A$, for $1 \leq i \leq k$.

Let $V^{n}$ denote the $n$-dimensional vector space $\mathbb{F}^{n}$ over $\mathbb{F}$, and denote by $V_{n}$ its dual vector space. Consider an element $f$ of the following 
tensor product space:

$$
f \in \bigotimes_{i=1}^{k}\left(V_{n_{i}}\right)^{\otimes_{n_{i}}}
$$

where $\left(V_{n}\right)^{\otimes_{n}}$ denotes the $n$-th tensor power of the vector space $V_{n}$. We denote by $f(\mathcal{A}) \in \mathbb{F}$ the evaluation of the multilinear form $f$ at the ordered set of column vectors of $\mathcal{A}$ (strictly speaking, one should write $f([\mathcal{A}])$ instead, but we simplify the notation by dropping the square brackets). Often in applications, one is interested in an alternating sum of the form

$$
\sum_{\sigma \in \Sigma} \operatorname{sgn}(\sigma) f\left(\sigma^{-1} \cdot \mathcal{A}\right)
$$

where

$$
\operatorname{sgn}(\sigma)=\prod_{i=1}^{k} \operatorname{sgn}\left(\sigma_{i}\right)
$$

We have the following theorem.

Theorem 2.1. If $\mathcal{A}=\left({ }^{1} A, \ldots,{ }^{k} A\right)$ is a $k$-tuple of square matrices and $f$ as above, then

$$
\sum_{\sigma \in \Sigma} \operatorname{sgn}(\sigma) f\left(\sigma^{-1} \cdot \mathcal{A}\right)=\mathcal{I}(f, \mathbf{n}) \operatorname{det}\left({ }^{1} A\right) \cdots \operatorname{det}\left({ }^{k} A\right)
$$

where $\operatorname{det}(A)$ denotes the determinant of a square matrix $A$, and $\mathcal{I}(f, \mathbf{n}) \in$ $\mathbb{F}$ is a scalar depending only on $f$ and on the shape $\mathbf{n}=\left(n_{1}, \ldots, n_{k}\right)$ of $\mathcal{A}$.

Corollary 2.2. As a corollary of the previous theorem, one can calculate $\mathcal{I}(f, \mathbf{n})$ by simply assuming that each ${ }^{i} A$ is an identity matrix, for $1 \leq i \leq k$. Namely, if we let

$$
I=\left(I_{n_{1}}, \ldots, I_{n_{k}}\right)
$$

where $I_{n_{i}}$ denotes the $n_{i}$-by- $n_{i}$ identity matrix, then

$$
\mathcal{I}(f, \mathbf{n})=\sum_{\sigma \in \Sigma} \operatorname{sgn}(\sigma) f\left(\sigma^{-1} . I\right)
$$

In applications, $f$ is often a determinant, or a product of determinants (or sum thereof), which represents a combinatorial problem for which the input data $\mathcal{A}$ has ambiguity, where the possible choices for $\mathcal{A}$ are usually parametrized by the elements of $\Sigma$. The scalar $\mathcal{I}(f, \mathbf{n})$ represents a combinatorial invariant of the problem. If $\mathcal{I}(f, \mathbf{n})$ is nonzero, then for any input data $\mathcal{A}$ (with ambiguity) for which all matrices ${ }^{i} A$ are non singular for $1 \leq i \leq k$ (we will simply say in this case that 
$\mathcal{A}$ is non-singular), the right-hand-side of $(2.3)$ is non-zero, which implies that one of the terms in the alternating sum on the left-hand side of (2.3) is non-zero. This translates into the statement that, if $\mathcal{I}(f, \mathbf{n}) \neq 0$, then given any non-singular input data $\mathcal{A}$ with ambiguity, a choice for $\mathcal{A}$ can be made so that some output matrix, or matrices, corresponding to $f$, are also non-singular. This is an outline of the general argument which will be made precise in the two applications we consider: Onn's colorful determinantal formula and Svrtan's $n$ ! formula, and their consequences.

Proof of Thm. 2.1. The (partial) skew-symmetrization of $f$ belongs to the following 1-dimensional space

$$
\sum_{\sigma \in \Sigma} \operatorname{sgn}(\sigma)(\sigma . f) \in \bigotimes_{i=1}^{k}\left(\Lambda^{n_{i}} V_{n_{i}}\right)
$$

where the action of $\Sigma$ on elements

$$
f \in \bigotimes_{i=1}^{k}\left(V_{n_{i}}\right)^{\otimes_{n_{i}}}
$$

is given by $(\sigma . f)(\mathcal{A})=f\left(\sigma^{-1} \cdot \mathcal{A}\right)$. But an element of $\Lambda^{n} V_{n}$ is a scalar multiple of the $n$-by- $n$ determinant, thought of as a multilinear form of the column vectors. We thus see, from equation $(2.5)$, that the alternating sum is some constant $\mathcal{I}(f, \mathbf{n})$ (constant meaning here independent of the values of the matrices $\left.{ }^{i} A\right)$ times the product of the determinants $\operatorname{det}\left({ }^{i} A\right)$, for $1 \leq i \leq k$. In other words, we have proved our main result, namely formula 2.3 .

\section{Onn's Colorful Determinantal Formula}

In this section, we assume that the ground field $\mathbb{F}$ is a field of characteristic 0 , and that $\mathcal{A}=\left({ }^{1} A, \ldots,{ }^{n} A\right)$, where each ${ }^{i} A$ is an $n$-by- $n$ matrix for $1 \leq i \leq n$. We denote by ${ }^{i} A^{j}$ the $j$-th column of the matrix ${ }^{i} A$. We define the multilinear form $f$ by

$$
f(\mathcal{A})=\prod_{i=1}^{n} \operatorname{det}\left({ }^{1} A^{i}, \ldots,{ }^{n} A^{i}\right)
$$

We introduce the group

$$
\left.\Sigma=\Sigma_{n} \times \cdots \times \Sigma_{n} \quad \text { (n factors }\right)
$$


where $\Sigma_{n}$ is the symmetric group on $[n]$. We write an arbitrary element $\sigma \in \Sigma$ as $\sigma=\left(\sigma_{1}, \ldots, \sigma_{n}\right)$. We then form the alternating sum

$$
\sum_{\sigma \in \Sigma} \operatorname{sgn}(\sigma) f\left(\sigma^{-1} . \mathcal{A}\right)=\sum_{\sigma \in \Sigma} \operatorname{sgn}(\sigma) \prod_{j=1}^{n} \operatorname{det}\left({ }^{1} A^{\sigma_{1}(j)}, \ldots,{ }^{n} A^{\sigma_{n}(j)}\right)
$$

By Thm. 2.1, we obtain that

$$
\sum_{\sigma \in \Sigma} \operatorname{sgn}(\sigma) f\left(\sigma^{-1} \cdot \mathcal{A}\right)=\mathcal{I}(f, n) \operatorname{det}\left({ }^{1} A\right) \cdots \operatorname{det}\left({ }^{n} A\right)
$$

It remains to calculate $\mathcal{I}(f, n)$ using Cor. 2.2. We thus assume that

$$
{ }^{i} A=I_{n} \quad(1 \leq i \leq n)
$$

where $I_{n}$ is the $n$-by- $n$ identity matrix. It can be shown that (cf. for instance [16] or [1])

$$
\mathcal{I}(f, n)=\sum_{\sigma \in \Sigma} \operatorname{sgn}(\sigma) \prod_{j=1}^{n} \operatorname{det}\left(e_{\sigma_{1}(j)}, \ldots, e_{\sigma_{n}(j)}\right)=l(n)
$$

where $l(n)$ is the number of even $n$-by- $n$ Latin squares minus the number of odd $n$-by- $n$ Latin squares. Combining the previous formula with formulas (3.1) and (3.2), we obtain Onn's colorful determinantal identity:

$$
\sum_{\sigma \in \Sigma} \operatorname{sgn}(\sigma) \prod_{j=1}^{n} \operatorname{det}\left({ }^{1} A^{\sigma_{1}(j)}, \ldots,{ }^{n} A^{\sigma_{n}(j)}\right)=l(n) \prod_{i=1}^{n} \operatorname{det}\left({ }^{i} A\right)
$$

The Alon-Tarsi conjecture is that $l(n) \neq 0$ if $n$ is even. Onn used his formula to show that the Alon-Tarsi conjecture implies Rota's basis conjecture for even $n$, which had been proved by Huang and Rota in [13. The argument is as follows. Assume $n$ is even and that the Alon-Tarsi conjecture is true, i.e. that $l(n) \neq 0$. If the matrices ${ }^{i} A$ are non-singular for all $i, 1 \leq i \leq n$, then the right-hand side of Onn's formula $(3.3)$ is non-zero, which implies that at least one of the terms in the alternating sum on the left-hand side of the same formula must be non-zero. But this is precisely the conclusion of Rota's basis conjecture.

\section{Svrtan's $n$ ! formula}

In this section, we choose to work over $\mathbb{C}$, though the results will all hold over any field $\mathbb{F}$ of characteristic 0 (or more generally any field in which $n$ ! is non-zero). Inspired by both Rota's basis conjecture, as well as the Atiyah-Sutcliffe problem on configurations of points, the author came up with a hybrid problem, which he initially posted on 


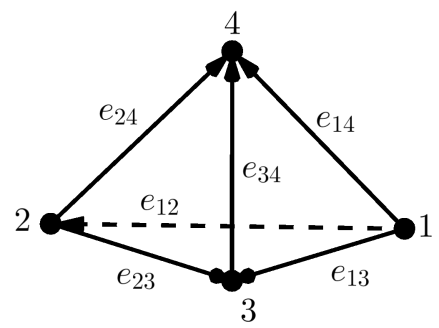

Figure 1. A representation of the graph $\Gamma_{4}$

an online forum (Math StackExchange at first, but then migrated to MathOverflow). The problem can be described as follows.

For $m$ a positive integer, denote by $V_{m}$ the space of complex polynomials of degree less than $m$ in one complex variable. Let $\Gamma_{n}$ be the complete oriented graph on $n$ vertices. The set of vertices may be taken to be $\{1, \ldots, n\}$, and the set of oriented edges becomes then $\{(i, j) ; 1 \leq i, j \leq n, i \neq j\}$. We will denote the oriented edge $(i, j)$ simply by $e_{i j}$. One may then introduce an equivalence relation $\sim$, where each $e_{i j}$ is equivalent only to itself and to $e_{j i}$. We denote the equivalence class of $e_{i j}$ by $\left[e_{i j}\right]$, and the quotient $\bar{\Gamma}_{n}$ of $\Gamma_{n}$ by $\sim$ is the non-oriented complete graph on $n$ vertices, whose set of (non-oriented) edges is $\left\{\left[e_{i j}\right] ; 1 \leq i<j \leq n\right\}$.

Assume there given, to each non-oriented edge $\left[e_{i j}\right], i<j$, of $\bar{\Gamma}_{n}$, an ordered basis $\left({ }^{i j} p_{1},{ }^{i j} p_{2}\right)$ of the space $V_{2}$. We call such an assignment an assignment of "spinor bases". We refer to $V_{2}$ as the space of spinors.

By an assignment $c$ of spinors corresponding to an assignment of spinor bases, we mean that to each $e_{i j}$, with $i<j$, we assign either ${ }^{i j} p_{1}$ or ${ }^{i j} p_{2}$, and we assume that once such a choice is made, the "other" choice is made for the edge $e_{j i}(i<j)$. So for instance, if to $e_{i j}$ we assigned ${ }^{i j} p_{2}$, we must then assign ${ }^{i j} p_{1}$ to $e_{j i}$. Once an assignment of spinors is made, we denote the spinor associated to each edge $e_{i j}(i \neq j)$ simply by $p_{i j}^{c}$, where $c$ refers to the assignment of spinors.

We denote by $\mathcal{C}$ the set of all possible assignments of spinors (corresponding to some fixed assignment of spinor bases), which contains exactly $2^{\left(\begin{array}{c}n \\ 2\end{array}\right)}$ elements. We define the $\operatorname{sign} \operatorname{sgn}(c)$ of an assignment $c \in \mathcal{C}$ of spinors to be 1 (resp. - 1 ) if the number of times ${ }^{i j} p_{2}$ was assigned to the edge $e_{i j}$ for $i<j$ is even (resp. odd). We say that an assignment of spinors $c$ is even (resp. odd) if $\operatorname{sgn}(c)=1$ (resp. $\operatorname{sgn}(c)=-1)$.

To each assignment $c \in \mathcal{C}$ of spinors, one forms $n$ complex polynomials $p_{i}^{c} \in V_{n}$, for $1 \leq i \leq n$, of degree less than $n$ in the complex variable 
$t$, obtained as follows. The polynomial $p_{i}$ is defined as:

$$
p_{i}^{c}=\prod_{j \neq i} p_{i j}^{c} \quad(1 \leq i, j \leq n)
$$

The problem can now be stated.

Problem: Given an arbitrary inital assignment of spinor bases $\left({ }^{i j} p_{1},{ }^{i j} p_{2}\right)$ to each non-oriented edge $\left[e_{i j}\right], i<j$, of the (non-oriented) complete graph $\bar{\Gamma}_{n}$ on $n$ vertices, does there always exist an associated assignment $c$ of spinors to each oriented edge $e_{i j}, i \neq j$, of the oriented complete graph $\Gamma_{n}$, for which the $n$ polynomials $p_{i}^{c}$, for $1 \leq i \leq n$, are linearly independent over $\mathbb{C}$ ?

Theorem 4.1. The answer to the previous problem is positive, namely, given any initial assignment of spinor bases to each (non-oriented) edge of $\bar{\Gamma}_{n}$, there always exists an associated assignment $c$ of spinors to each (oriented) edge of $\Gamma_{n}$, for which the associated $n$ polynomials $p_{i}^{c}$, for $1 \leq i \leq n$, are linearly independent over $\mathbb{C}$.

Svrtan's theorem [15, pp. 21-23], slightly modified to be adapted to our notation, can be written as follows:

Theorem 4.2 (Svrtan's $n$ ! Formula).

$$
\sum_{c \in \mathcal{C}} \operatorname{sgn}(c) \operatorname{det}\left(p_{1}^{c}, \ldots, p_{n}^{c}\right)=n ! \prod_{1 \leq i<j \leq n} \operatorname{det}\left({ }^{i j} p_{1},{ }^{i j} p_{2}\right)
$$

Proof of Surtan's Theorem. The proof follows from Thm. 2.1, with $\mathcal{A}=\left(\left({ }^{i j} p_{1},{ }^{i j} p_{2}\right) ; 1 \leq i<j \leq n\right)$, and

$$
f(\mathcal{A})=\operatorname{det}\left(p_{1}^{c_{0}}, \ldots, p_{n}^{c_{0}}\right)
$$

where $c_{0} \in \mathcal{C}$ corresponds to assigning ${ }^{i j} p_{1}$ to each $e_{i j}, 1 \leq i<j \leq n$ ( $c_{0}$ is thus even). Indeed formula (2.3) then implies

$$
\sum_{c \in \mathcal{C}} \operatorname{sgn}(c) \operatorname{det}\left(p_{1}^{c}, \ldots, p_{n}^{c}\right)=\mathcal{I}(f, n) \prod_{1 \leq i<j \leq n} \operatorname{det}\left({ }^{i j} p_{1},{ }^{i j} p_{2}\right)
$$

It remains only to check that $\mathcal{I}(f, n)=n$ !. In order to calculate $\mathcal{I}(f, n)$, it suffices by Cor. 2.2 to assume that

$$
\left\{\begin{array}{l}
{ }^{i j} p_{1}=1 \\
{ }^{i j} p_{2}=t
\end{array}\right.
$$

$(1 \leq i<j \leq n)$. Given any (non-oriented) edge $\left[e_{i j}\right](i<j)$ of $\bar{\Gamma}_{n}$, we orient it (i.e. assign to it a direction) from $i$ to $j$ (resp. from $j$ to $i)$ if ${ }^{i j} p_{1}=1$ is assigned to $e_{i j}\left({ }^{i j} p_{2}=t\right.$ is assigned to $\left.e_{i j}\right)$. We 
see that there must be exactly one vertex having exactly $r$ outgoing vertices, for every $r$ with $0 \leq r \leq n-1$. Therefore only $n$ ! terms are non-zero in the alternating sum on the left-hand side of (4.1), the ones corresponding to some complete order $\prec$ on the set $[n]=\{1, \ldots, n\}$, with the corresponding assignment of spinors being

$$
i \prec j \Longrightarrow{ }^{i j} p=1
$$

More precisely, $\operatorname{det}\left(p_{1}^{c}, \ldots, p_{n}^{c}\right)=1$ (resp. -1) if $c$ corresponds to a complete order $\prec$ which in turn corresponds to an even (resp. odd) permutation of $[n]$. We therefore deduce that

$$
\mathcal{I}(f, n)=\sum_{c \in \mathcal{C}} \operatorname{sgn}(c) \operatorname{det}\left(p_{1}^{c}, \ldots, p_{n}^{c}\right)=n !
$$

corresponding to the assignment of spinor bases in (4.3). Svrtan's formula then follows, using equation (4.2).

We can now prove Thm. 4.1. Indeed, given an assignment of spinor bases, it is clear that the right-hand side of Svrtan's $n$ ! formula (4.1) is non-zero, so that one of the terms in the alternating sum on the left-hand side of the same formula must be non-zero. This implies that there is some associated assignment $c \in \mathcal{C}$ of spinors, for which the polynomials $p_{1}^{c}, \ldots, p_{n}^{c}$ are linearly independent over $\mathbb{C}$, proving the theorem.

Acknowledgements. The author thanks Timothy Chow for an interesting email exchange in which Dr. Chow informed the author about some of the literature surrounding Rota's basis conjecture, and suggested a line of attack similar to one initiated by Rota himself and applied to his conjecture, which had led to a link between his conjecture and the Alon-Tarsi conjecture concerning the number of even/odd latin squares of order $n$ (cf. [13] for the original work, and [14 for a nicely written alternative short argument). The author is also indebted to the anonymous referee, who in particular provided a simpler proof of Svrtan's $n$ ! formula than the author's original proof, and this inspired the author to find a more general formula, using a similar idea.

\section{References}

[1] Aharoni, R. and Loebl, M., The odd case of Rota's bases conjecture, Adv. Math. 282 (2015), 427-442.

[2] Atiyah, M. F., The geometry of classical particles, Surveys in differential geometry, 1 - 15, Surv. Differ. Geom., VII, Int. Press, Somerville, MA, 2000. 
[3] Atiyah, M. F., Configurations of points, Topological methods in the physical sciences (London, 2000). R. Soc. Lond. Philos. Trans. Ser. A Math. Phys. Eng. Sci. 359 (2001), no. 1784, 1375-1387.

[4] Atiyah, M. F. and Malkoun, J., The Relativistic Geometry and Dynamics of Electrons, Found. Phys. 48:2 (2018), 199-208.

[5] Atiyah, M. F. and Sutcliffe, P. M., The geometry of point particles, R. Soc. Lond. Proc. Ser. A Math. Phys. Eng. Sci. 458 (2002), no. 2021, 1089-1115.

[6] Berry, M. V. and Robbins, J. M., Indistinguishability for quantum particles: spin, statistics and the geometric phase, Proc. Roy. Soc. London Ser. A 453 (1997), 1771-1790.

[7] Bollen, G. P. and Draisma, J., An online version of Rota's basis conjecture, J. Algebraic Combin. 41 (2015), 1001-1012.

[8] Chow, T., On the Dinitz conjecture and related conjectures, Disc. Math. 145 (1995), 73-82.

[9] Chow, T., Fan, C. K., Goemans, M. X. and Vondrak, J., Wide partitions, Latin tableaux, and Rota's basis conjecture, Advances Appl. Math. 31 (2003), 334358.

[10] Chow, T., Reduction of Rota's basis conjecture to a conjecture on three bases, Siam J. Disc. Math. 23 (2009), 369-371.

[11] Geelen, J. and Humphries, P., Rota's basis conjecture for paving matroids, SIAM J. Discrete Math. 20 (2006), no. 4, 1042-1045.

[12] Geelen, J. and Webb, K., On Rota's basis conjecture, SIAM J. Discrete Math. 21 (2007), 802-804.

[13] Huang, R. and Rota, G.-C., On the relations of various conjectures on Latin squares and straightening coefficients, Discrete Math. 128 (1994), 225-236.

[14] Onn, S., A colorful determinantal identity, a conjecture of Rota, and Latin squares, Amer. Math. Monthly 104 (1997), 156-159.

[15] Svrtan, D., A Proof of All Three Euclidean Four Point Atiyah-Sutcliffe Conjectures, https://www.emis.de/journals/SLC/wpapers/s73vortrag/svrtan. pdf.

[16] Zappa, P., The Cayley Determinant of the Determinant Tensor and the AlonTarsi Conjecture, Adv. Appl. Math. 19 (1997), 31-44

Department of Mathematics and Statistics, Faculty of Natural and Applied Sciences, Notre Dame University-Louaize, Zouk Mosbeh, P.O.Box: 72, Zouk MiKAEL, LEBANON

E-mail address: joseph.malkoun@ndu.edu.lb 